\title{
Disaster Recovery Power and Communications for Smart Critical Infrastructures
}

\author{
Abdulaziz Alqahtani ${ }^{1}$, Rohit Abhishek ${ }^{2}$, David Tipper ${ }^{1}$ and Deep Medhi ${ }^{2}$ \\ ${ }^{1}$ School of Computing and Information, University of Pittsburgh, Pittsburgh, PA \\ ${ }^{2}$ School of Computing and Engineering, University of Missouri - Kansas City, Kansas City, MO
}

\begin{abstract}
In this paper, we propose a framework to leverage electrical microgrids and cellular networks to support postdisaster communications for the public, government and critical infrastructure operation. The framework involves both policy and technical components. The proposed approach is an integration of electrical microgrids to provide power together with self configuring wireless mesh communication networks and local edge computing infrastructure to support critical communications and smart infrastructure services/applications in a specific geographic area. Hence, geographic zones which are resilient safe havens are created in a city. We outline the basic components of our approach and discuss open challenges to realizing the vision.

Index Terms-Disaster Recovery, Cellular Networks, Microgrids, Smart Cities
\end{abstract}

\section{INTRODUCTION}

Cellular phone communications have become ubiquitous in the developed world and are part of the critical infrastructures upon which society depends. Furthermore cellular communication networks are being viewed as a key communication component for smart city and Internet of things (IoT) applications (e.g.,using LTE-M or NB-IoT). The combination of advances in sensors, communication networks, controls, cyberphysical systems, data integration and analytics is leading to smart infrastructures for smart cities by improving sustainability, efficiencies, and the quality of life of residents. Numerous smart city systems have been proposed and testbeds have been deployed in areas such as improving energy efficiency, transportation systems, environmental monitoring and asset management [1], [2]. Some specific examples are smart water systems using wireless sensors and actuators installed in/along water distribution networks to monitor pressure, leaks and ruptures, water quality and optimize the flow [3] and integrated sensors, traffic control and public transportation for intelligent transportation systems [4]. As research and development continues in the smart city infrastructure space, many new applications are expected to emerge.

Note that these new smart infrastructures are increasingly dependent on both electrical power and ICT. This creates new interdependencies and vulnerabilities, especially to natural disasters and extreme weather (e.g, hurricanes, tornados, wind storms, ice storms, etc.). Recent studies [5] show that weather events are the main cause of large power outages in the United States and, in turn, power outages are a leading cause of downtime in cellular communication networks. Furthermore, the size and the number of power outages caused by weather events is expected to rise as climate change increases the intensity and frequency of extreme weather (i.e., hurricanes, floods, blizzards, tornados, etc.) [6]. For example, according to the US government, the annual rate of weather related events having an economic impact of 1 billion or more, averaged 10.6 events during the time period 2012 to 2016, which is a sharp increase from the long term average of 5.64 events per year over 1980 to 2016. Such extreme weather events typically result in long power outages lasting days or even weeks severely impacting society in general. This is illustrated by the recent 2017 hurricane Maria impact on the island of Puerto Rico, where electrical power, cellular communications and water distribution systems were impacted for weeks.

In general, cellular communications networks have proven to be fragile in the face of natural disasters leading to recent work on post disaster networks [7] and techniques to make networks more robust to disasters [8]. However, theses works primarly concentrate on the communication network in isolation, ignoring the need for power or assuming temporary solutions (e.g., batteries, cells on wheels) are sufficient. Thus far, little work has appeared on improving the survivability and resilience of critical infrastructure in a smart city context. In this paper, we propose a framework to address the problem of providing resilient power and ICT to support smart infrastructure applications under natural disaster conditions. Our approach is a combination of a multi-user electrical microgrid to provide power together with cellular based communications dynamically reconfigured into a mesh network and local edge computing resources to support critical smart infrastructure services/applications in a specific geographic area. The goal is to create geographic districts within a city that are safe havens with critical services functioning at a degraded but acceptable level of service in the face of extreme conditions.

The rest of the paper is organized as follows. In Section II, we present our proposed architectural framework followed by details on microgrids, cellular based wireless mesh networks and support for the smart infrastructures during disaster scenarios. Section III presents results on microgrid costs. Lastly, section IV presents our conclusions and future work.

\section{RESILIENT POWER AND COMMUNICATIONS FRAMEWORK}

We consider a scenario where a natural disaster has resulted in a power outage of size and duration such that commercial 
cellular networks have outages and the smart infrastructures which depend on a steady supply of electricity as well as cellular communication services are adversely affected. The cellular network outage maybe due to the failures of base stations and/or the backhaul network and/or associated core network services (e.g., authentication, mobility management, synchronization, etc.). Note, that while some cell sites may have backup batteries (typically 4 to 8 hours of power) or diesel gensets, they cannot provide service without backhaul network connectivity and core network services (this was observed in 2012 hurricane Sandy in New Jersey where powered base stations could not provide service due to flooded backhaul equipment resulting in isolation from the core network). Here we propose to use edge computing devices together with dynamic reconfiguring of powered cellular network base stations across operators including pooling the available spectrum to form a multihop ad hoc mesh network which can provide local disaster communication services to the public, government and smart infrastructures.

The components of our framework are illustrated in Figure 1. There are two major pieces: (1) a multi-user microgrid and (2) a disaster recovery cellular based communication network that is organized into a multihop wireless mesh network. We discuss them in turn below.

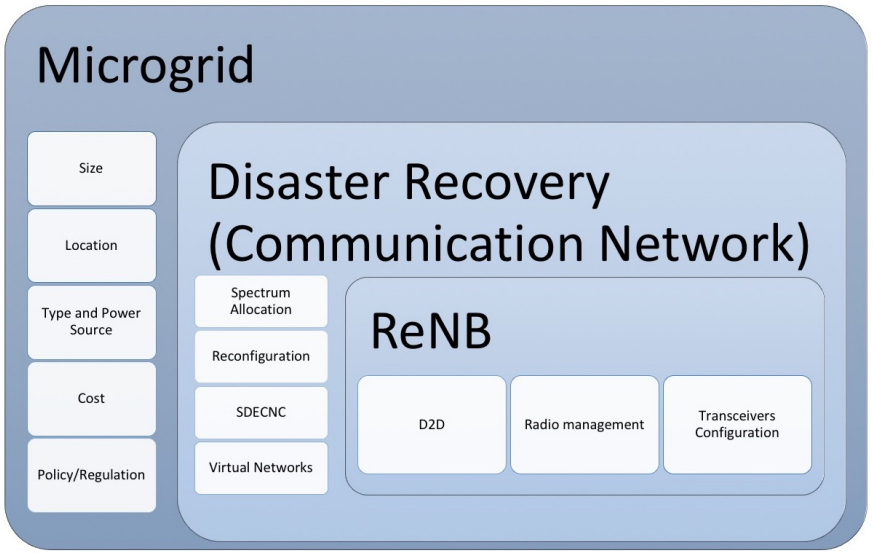

Fig. 1. Architecture Framework

\section{A. Microgrids}

Microgrids are essentially small scale power systems usually on the medium to low voltage distribution feeder that include distributed generation together with protection devices and possibly energy storage [9]. Microgrids have been proposed as a method to provide continuity of power to key commercial and societal locations, (e.g., military installations, hospitals). Also, microgrids have been touted as a mechanism to facilitate the incorporation of distributed renewable energy generation, such as solar and wind, into the grid and modernize the power grid in an incremental fashion. The functional building blocks of microgrids include the electrical switches and protection to connect to/from the main power grid, interconnected electrical loads and a local energy supply (e.g., fuel cells, renewables, diesel gensets).

The basic requirements for microgrids are to operate in a stand-alone mode (i.e., the so called island mode) and a main grid connected mode. In the island mode, the microgrid operates on local sources of energy with the local microgrid control system providing voltage and frequency (in the case of AC) stability for optimal power flows, and ensuring minimal load shedding and disruption during transitions from the main power grid connected to the island mode.

Figure 2 shows a high level view of a microgrid, illustrating the local power sources and the electrical loads, which are grouped into classes based on their importance, namely, mission critical loads, mission priority loads, and non-critical loads. Note, the power generated or stored in a microgrid must equal the electrical load, and hence, depending on the power available in island mode, some classes of load may not be served, such as, non-critical loads and some mission priority loads. Further, the microgrid must have the ability to transition back from the island mode to main grid connected mode, resulting in re-synchronization with minimum impact to critical loads during the mode transition periods. In the connected mode, the microgrid acts as a supplemental power source to the main grid. Here we adopt the US Department of Energy's definition of a microgrid in that it is a single controllable entity with respect to the main power grid.

As listed in Figure 1, microgrids have several defining characteristics. First is the size of the microgrid in terms of power generation and storage. The majority of current and proposed industrial size microgrids are in the $1.5-40 \mathrm{MW}$ range. Traditionally microgrids have a fixed boundary and are designed to provide sufficient power to support mission critical loads within the geographic boundary utilizing their local generation and storage facilities. If the microgrid has surplus power available, less important loads can be powered or the microgrid can act as a virtual feeder picking up nearby mission critical loads outside the boundary [10]. However, the virtual feeder operation requires determining feasible paths to connect to the load using automated feeder switches. In addition to the size of the microgrid, the geographic location of the microgrid and the loads it must power to enable specific specific smart city applications must be determined. In general the microgrid power source and energy storage should be located close to the loads in order to minimize the distribution line loss.

Another characteristic of microgrids is the type of power generated (i.e., $\mathrm{AC}, \mathrm{DC}$ or mix of $\mathrm{AC}$ and $\mathrm{DC}$ ), how the power is distributed (AC or DC) and the associated load requirements (AC, DC or mix). For example, some renewables energy sources, such as solar, produce DC power that must be converted to $\mathrm{AC}$ for $\mathrm{AC}$ loads. In general, conversion (e.g., $\mathrm{AC} / \mathrm{DC}$ ) is inefficient due to power losses and should be minimized. Regulatory policies are a major issue in regards to microgrids as they can restrict ownership, confine the type of connection to the main grid and limit the power generation size. For example, some states forbid the local utility from owning and operating microgrids and put limits 


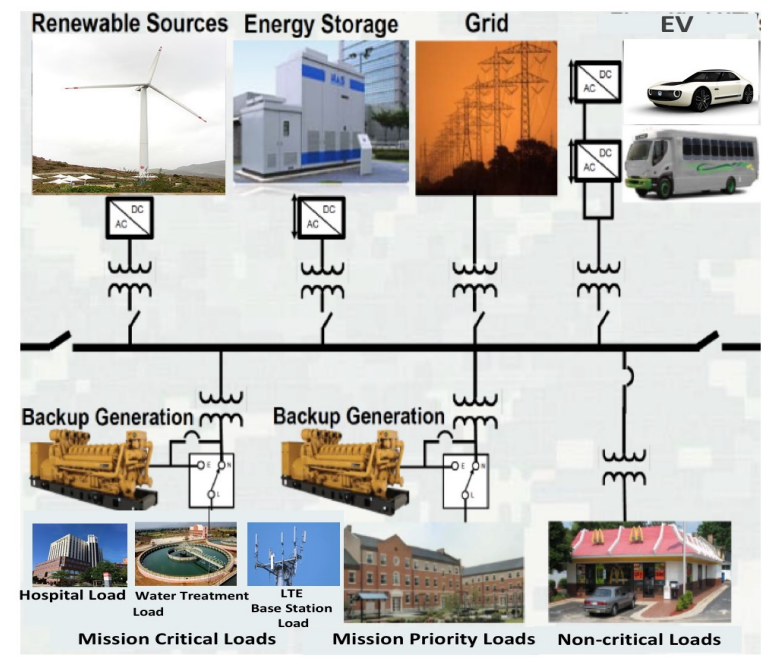

Fig. 2. Microgrid Architecture

on the maximum size of microgrids to avoid regulation.

A major hurdle to the deployment of microgrids are the economic barriers in terms of cost to own and operate an industrial scale microgrid. Traditionally microgrids are owned and operated by a single entity. In [11], the authors propose a resilient DC microgrid using renewable energy (wind + solar) to power a cluster of nearby LTE cellular base stations. However, the cost of tens of small microgrids just to power a cellular network throughout a city during power outages is prohibitive. Given the non-linear economics of microgrids[12], we propose mult-user microgrids be deployed such that the cost is shared by the smart infrastructure owners with mission critical loads (e.g., water system, cellular networks), societal important locations with mission critical loads (e.g., hospital), and the local government (police, fire, 911 service) that will utilize the communication network powered by the microgrid for public safety communications. This would provide government sponsored options for financing industrial sized microgrids, but may require regulatory changes in many juristrictions to enable multi-user microgrids.

\section{B. Communication Network}

The other component in the framework of Figure 1 is the disaster recovery communication network. In most large cities, there are multiple LTE cellular network operators with overlapping coverage as shown in Figure 3 with two operators. We propose to leverage this communication network landscape to enable smart infrastructures to communicate between their mission critical components in order to maintain full or partial operation. Further, we intend to size and locate the microgrid so that it can power both mission critical smart infrastructure components and a set of geographically nearby base stations.

Note, the LTE network architecture can be divided into two main parts namely: (1) the access network and (2) the core network. The access network consist of the base stations termed evolved NodeBs (eNBs) in LTE. The core network consists of a variety of components, namely: mobility management entity (MME), serving gateway (S-GW), packet gateway (P-GW), policy charging rules function (PCRF), and home subscriber service HSS). The core network component provide services related to mobility, security, billing, addressing and connection to data and circuit-switched networks. Cellular networks are not particularly robust since if the access portion of the network is disconnected from the core network, the access network is unable to provide service. Hence, even if the base stations are undamaged and are supplied power from the microgrid after a disaster event, they will not be able to provide service if they have lost backhaul connection to the core network or core network is down due to the disaster event.

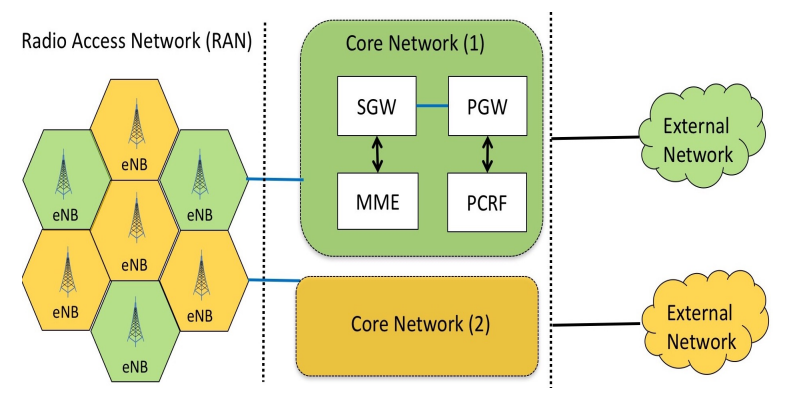

Fig. 3. Typical cellular network topology

In this work, we propose to form a software defined network (SDN) using any undamaged eNB base stations in order to maintain LTE based local communication service to the user equipments (UEs) and the LTE-based smart infrastructure sensor/machines within range. The basic idea is to reconfigure the base stations powered by the microgrid to form a multihop wireless mesh network (WMN) as illustrated in Figure 4. The basic components needed to form the WMN are shown in Figure 1 and are discussed below.

1) Reconfigurable-eNB: In order to form the WMN, we propose to modify the eNB base stations to Reconfigurable$e N B$ (ReNB) that have the ability to create a local serving radio access network without backhaul communications to the core network. The ReNB will be equipped with SDN functionality and multiple radio transmitters to support the creation of radio links between neighboring base stations, which maybe owned by different network operators. The ReNB will need to support radio management (i.e., MIMO configurations, power levels, etc.) of the links between base stations as well as links to UEs within coverage range. Also, the ReNB should support direct device-to-device (D2D) communication [13] in order to extend the cellular coverage through multiple UE hops to the ReNB. Similarly, D2D can be used to extend the cellular coverage through multiple UE hops for smart infrastructures components that are within range of each other.

2) Spectrum Allocation: Consider that a subset of cellular network base stations from different operators on different frequency bands maybe be powered within the microgrid boundary. In the absence of the backhaul connections, we propose to pool the available licensed spectrum bands and 
reallocate the spectrum to support the wireless mesh network. In particular, some spectrum bands are used to support communication links between ReNB base stations, other bands are allocated to optimize coverage and performance. For example, Figure 4 shows a WMN powered by a microgrid. The shaded rectangular area denotes the geographic boundary of the microgrid and the lines between base stations indicate point to point wireless links. The base stations are a mix of small and large cells and may include new technology such as LTE-U and small $5 \mathrm{G}$ cells. If the larger base stations in the WMN are assigned higher frequency band spectrum for serving users (e.g., $1.9 \mathrm{GHz}$ ), then the coverage area would be the area indicated by the red cloud. However, if a lower spectrum band (e.g., 700MHz) is assigned to the larger base stations, the geographic coverage area will be larger as shown by the blue cloud in the figure. Note that in both cases, the radio coverage area is larger than the geographic boundary of the microgrid, and thus, the network can provide service to devices outside the microgrid boundary if those devices have sufficient battery power or a local power source. This is especially important for public safety applications using cell phones.

In general, there are several options for pooled spectrum allocation among the base stations. This can be done on the basis of specific preplanned scenarios, or a dynamic assignment can be made based on the scenario in hand (e.g., which base stations are operable, interference levels, capabilities of base stations, etc.). In order for the concept of spectrum pooling and reallocation of licensed spectrum to be viable, government policy and regulation must support this from a legal standpoint for disaster situations. Besides the licensed spectrum, we propose to also use unlicensed spectrum with technologies such as $L T E-U, L A A$, and IEEE $802.11 \mathrm{WiFi}$ offloading when possible.

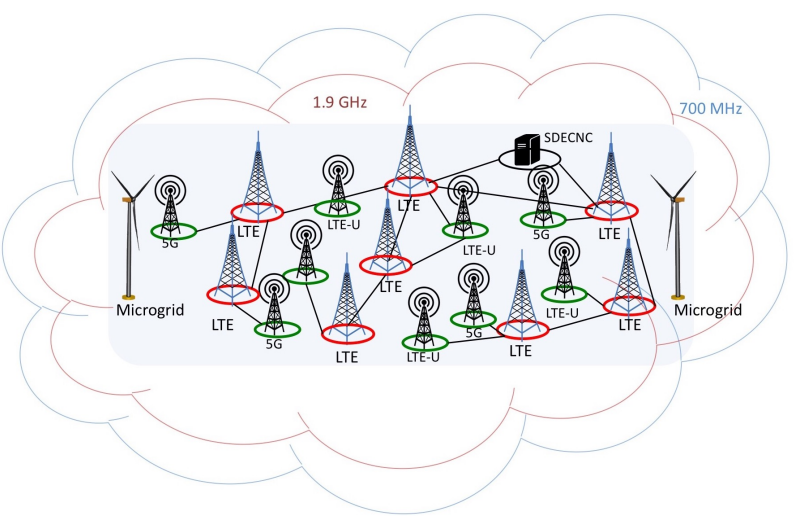

Fig. 4. Cellular Mesh Network

3) Reconfiguration: One of the main functions of the ReNB is the ability to dynamically form a wireless mesh network during a disaster event. Rather than embed all the core network functions in the ReNB which would be cost prohibitive, we propose adding the minimum functions to the ReNB to form communication channels and routing in the case of emergencies. All other functions will be implemented into a specific server that we name the Software Defined Emergency Communication Network Controller (SDECNC). When the ReNB moves to the emergency mode, it will utilize a network reconfiguration protocol. The reconfiguration protocol supports the discovery of neighbor nodes and the establishment of radio links between neighbors forming a mesh network and contacting the SDECNC. A number of neighbor discovery protocols exists that could be adopted or modified for this use. The basic procedure involves scanning frequencies for a beacon from other nodes and responding, or failing that, transmitting a beacon/hello signal followed by listening for a response followed by performing a handshake and the frequency assignment to establish a link. Once the ad hoc network has been formed, a routing protocol will be needed for connections that span multiple wireless hops. This routing protocol would be installed using the SDECNC which will act as the SDN controller. There are several routing protocols in the literature for wireless mesh networks that can be adopted here, such as AODV (Ad hoc On-Demand Distance Vector), Associativity-Based Routing (ABR) [14], and B.A.T.M.A.N. (Better Approach To Mobile Adhoc Networking). Note that the UE should preceive the service as standard LTE service in normal forms (i.e., unicast, multicast, broadcast) and does not need any special equipment or software.

4) Software Defined Emergency Communication Network Controller (SDECNC): The SDECNC will act as the SDN controller for the ad-hoc network formed by the ReNBs. Once the ReNB discovers all its neighbors, it advertises them to all its neighbors (similar to Link State Advertising). Simultaneously an the SDECNC controller will send a LSA to all its neighbors about it being assigned the controller. The controller by means of LSA will know about the neighbors to which each ReNB can be connected by establishment of a link. The use of SDN concepts eliminates the creation of unnecessary links between the different ReNBs. The SDECNC is a server that encompasses additional functions usually performed by the core network and other auxiliary services. For example, the SDECNC would perform authentication/security management, timing services such as PTP based clock synchronization for ReNBs, mobility management, PSAP processing, DNS, etcetra. The SDECNC requires a steady supply of power and a wired or wireless connectivity to some base stations in the WMN. Hence, the ENCS should be located within the geographic boundary of the microgrid. The SDECNC will maintain control signaling connections to ReNB and UEs in a fashion to the S1-MME connections in LTE.

5) Virtual Networks : A virtual network (VN) refers to a class of service that uses a shared network infrastructure to emulate the characteristics of a private network. The virtual network must appear to the users as a fully functional network. The primary motivation behind implementing VNs is to obtain different service levels such as bandwidth, security, QoS, or availability. In [15], a framework was proposed to prioritize network traffic adaptively for smart cities using a softwaredefined network (SDN) approach, where services that require 


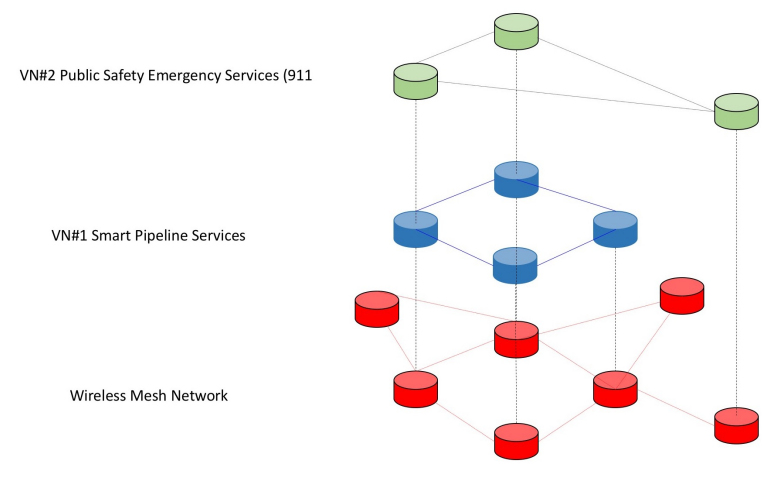

Fig. 5. Virtual Networks

priority are placed in virtualized networks and the mechanism is accomplished through a priority management layer in the SDN architecture. Here, we suggest the use of VNs in the wireless mesh network in order to prioritize and tailor services to different smart infrastructure and smart city applications. The services will be pre-classified according to the importance of the VN application. The SDECNC will act as the SDN controller here. For example, in Figure 5, public safety communication and smart infrastructure communications are each given a dedicated virtual network. The public safety communication virtual network VN2 can be designed to have higher reliability and fault tolerance than normal communications over the WMN, whereas, virtual network VN1 supporting smart water system communications is given real-time delay guarantees.

\section{Microgrid Evaluation}

In this section, we demonstrate how a multi-user microgrid sharing power over critical smart infrastructures can be costeffective. Here, we study microgrid cost sharing within a geographic area that has a hospital, water treatment plant and LTE base stations. First, we start by determining the power consumption for each infrastructure, beginning with the hospital. According to Schneider Electric [16], annual energy consumption can be found by using the number of beds in the hospital $(K W h / B e d)$ as follows:

$$
P_{H}=N * \overline{U_{X}}(k W h)
$$

where, $P_{H}$ is the power consumption for hospital $X$ with $N$ beds and $\overline{U_{X}}$ is the average bed power consumption in $k W h$. In this case, we consider a hospital with total bed size of 300 , which will result in overall $24000 \mathrm{kWh} / \mathrm{d}$ in power consumption.

We consider a water treatment plant that cleans seawater or brackish river/lagoon water for consumer use. According to American Authority in Membrane Treatment [18], the minimum amount of energy required to desalinate average seawater is approximately 3.8 kilowatt-hours per thousand gallons $(\mathrm{kWh} / \mathrm{kgal})$. Therefore, we can estimate the average power consumption of a water treatment as follows:

$$
P_{W}(k W h)=W_{X}(\text { gallon }) / 1000 * 3.8(k W h / k g a l)
$$

where, $W_{X}$ is the total water processed. Here, we consider a medium size city of population 300,000 that consumes 22.5 million gallons of water per day.

For LTE base stations, we use the average annual power consumption per base station as given in [19] as $\approx 8000 \mathrm{kwh} / \mathrm{d}$. Table I summarizes the power consumption for the infrastructures.

We utilized the HOMER microgrid simulation [?] and design software to estimate microgrid costs. In each case we designed a single line diagram microgrid that includes a mix of diesel generators, DC/AC converters, photovoltaic cells, wind turbines, and $1 \mathrm{KW}$ lithium acid batteries for storage. We considered powering each infrastructure with an individual microgrid and compared that to a single multi-user microgrid that can power all three infrastructures. Table II shows the result for this simulation; the first column reflects total load for each infrastructure. The second column indicates the net present cost, which includes (capital, replacement, salvage, operating and maintenance, and fuel) with project lifetime of 25 years.

The main parameters to be included here is the cost of energy, and by multiplying that with the whole electricity consumption, we measure the total power cost for each infrastructure as shown in the last column in the table.

By adding the total cost of each infrastructure that powered by a separate microgrid and compares it with the total value of each infrastructure that all powered by one microgrid, we noticed that the sharing one significant microgrid components are preferred in term of cost compare to the stand-alone small individual microgrid.

TABLE I

POWER CONSUMPTION

\begin{tabular}{|c|c|c|c|}
\hline Result & Hospital & Water Plant & LTE Base stations \\
\hline \hline Size & 300 beds & 8 million Gallon & 10 \\
\hline Power $(\mathrm{kWh} / \mathrm{d})$ & 24000 & 30000 & 8000 \\
\hline
\end{tabular}

TABLE II

SiMULATION RESULT

\begin{tabular}{|ccccccc|}
\hline \multicolumn{7}{|c|}{ Pittsburgh, PA } \\
\hline \hline & Load (kwh/d) & NPC & COE & OC & power kwh/y & Cost/y \\
\hline LTE BSs & 8000 & 16 & 0.425 & 1.1 & 2920000 & 1241000 \\
Hospital & 24000 & 46 & 0.419 & 3.5 & 8760000 & 3670440 \\
WWTP & 30000 & 58 & 0.416 & 4.2 & 10950000 & 4555200 \\
Total sharing & 62000 & 117 & 0.411 & 8.7 & 22630000 & 9300930 \\
Total Non-sharing & 62000 & 120 & - & 8.8 & 21389000 & 9466640 \\
\hline
\end{tabular}

Next we increased the number of base stations powered by the microgrid in steps of ten from 10 to 40 in figure 6 . The graph shows the different in cost between the sharable power source and non-sharable power source. Observe that the shared microgrid is always preferred over using a stand-alone power source for each load since result in less cost.

Next we increased the number of base stations powered by the microgrid in steps of ten from 10 to 40 in figure 6 . The 
Cost $\$ / k W h$

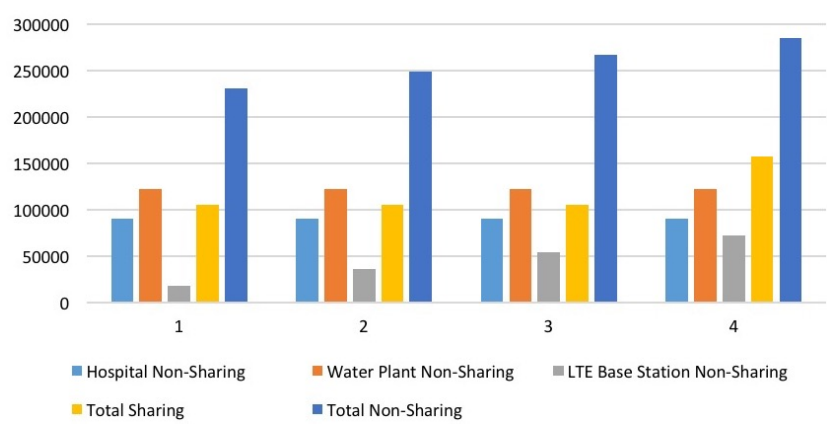

Fig. 6. Power Cost

graph shows the different in cost between the sharable power source and non-sharable power source. Observe that the shared microgrid is always preferred over using a stand-alone power source for each load since result in less cost.

\section{A. Smart Infrastructures}

As noted early the purpose of our proposed framework is to provide power and communications support to smart infrastructures such as water systems so that they may continue to operate in a district of a city. This requires identifying the mission critical electrical loads for the infrastructure and the necessary ICT support for operation. The mission critical loads can be determined by examining the infrastructure in question and can be connected to the microgrid for power. In terms of the ICT support the WMN can provide connectivity within the geographic boundary of the microgrid and nearby locations. However smart infrastructures will typically include a control and back end data analytic center. Hence either the some mission critical portion of the control and back end data analytic center functions must be provided by a local edge computing center located within the microgrid boundary or the WMN must provide connectivity to the main center which should have it's own power source. Determining what functions should be provided locally or the feasibility of connection to the normal control center will depend on the particular infrastructure and is one area of our future work.

\section{CONClusions}

In this paper, we propose a framework to provide power and communications support to smart city critical infrastructures. The framework advocates the use of microgrids to power smart city critical infrastructures within a specific district of a city. Furthermore, we propose to use the microgrid to power the cellular network base stations located within the microgrid boundary and reconfigure them into a standalone wireless mesh network that operates in a multi-hop fashion. This is facilitated by pooling different operators spectrum bands to be shared in case of emergencies and the use of an emergency communication network server to provide core network functions. We also proposed a reconfigurable eNB that has the capabilities to communicate with other ReNBs to create the wireless mesh network and the use of SDN based virtual networks to seperate different services/users and tailor quality of service. A detailed analysis of the ad hoc wireless mesh network and where to locate the microgrid to provide the greatest benefit to a city are future work topics.

\section{REFERENCES}

[1] S. P. Mohanty, U. Choppali, and E. Kougianos, "Everything you wanted to know about smart cities: The internet of things is the backbone," IEEE Consumer Electronics Magazine, vol. 5, no. 3, pp. 60-70, July 2016.

[2] A. Arroub, B. Zahi, E. Sabir, and M. Sadik, "A literature review on smart cities: Paradigms, opportunities and open problems," in Wireless Networks and Mobile Communications (WINCOM), 2016 International Conference on. IEEE, 2016, pp. 180-186.

[3] (2017, October 30 2017) Swan,the smart water networks forum. [Online]. Available: https://www.swan-forum.com/

[4] M. Tubaishat, Q. Qi, Y. Shang, and H. Shi, "Wireless sensor-based traffic light control," in 2008 5th IEEE Consumer Communications and Networking Conference, Jan 2008, pp. 702-706.

[5] US Department of Energy, "Economic benefits of increasing electric grid resilience to weather outages," http://energy.gov/sites, Aug, 2013.

[6] National Centers for Environmental Information (NCEI), "Billion-dollar weather and climate disaster: overview," [Online] http://www.ncdc.noaa.gov/billions, July, 2016.

[7] K. Miranda, A. Molinaro, and T. Razafindralambo, "A survey on rapidly deployable solutions for post-disaster networks," IEEE Communications Magazine, vol. 54, no. 4, pp. 117-123, 2016.

[8] T. Gomes, J. Tapolcai, and e. a. Esposito, Christan, "A survey of strategies for communication networks to protect against large-scale natural disasters," in 8th International Workshop on Resilient Network Design and Modeling (RNDM 2016). IEEE, Sept., 2016, pp. 11-22.

[9] X. Wang, A. V. Vasilakos, M. Chen, Y. Liu, and T. T. Kwon, "A survey of green mobile networks: Opportunities and challenges," Mobile Networks and Applications, vol. 17, no. 1, pp. 4-20, 2012. [Online]. Available: http://dx.doi.org/10.1007/s11036-011-0316-4

[10] Y. Wang, C. Chen, J. Wang, and B. Baldick, "Research on resilience of power systems under natural disasters," IEEE Transactions on Power Systems, vol. 31, no. 2, pp. 1604-1613, 2016.

[11] Y. Kwon, A. Kwasinski, and A. Kwasinski, "Coordinated energy management in resilient microgrids for wireless communication networks," IEEE Journal of Emerging and Selected Topics in Power Electronics, vol. 4, no. 4, pp. 1158-1173, 2016.

[12] R. Hanna, M. Ghonima, J. Kleissl, G. Tynan, and D. Victor, "Evaluating business models for microgrids: Interaction of technology and policy," Energy Policy, vol. 103, no. 4, pp. 47-61, 2017.

[13] A. Asadi, Q. Wang, and V. Mancuso, "A survey on device-to-device communication in cellular networks," IEEE Communications Surveys Tutorials, vol. 16, no. 4, pp. 1801-1819, Fourthquarter 2014.

[14] C. K. Toh, Ad Hoc Wireless Networks: Protocols and Systems, 1st ed Upper Saddle River, NJ, USA: Prentice Hall PTR, 2001.

[15] R. Abhishek, S. Zhao, and D. Medhi, "Spartacus: Service priority adaptiveness for emergency traffic in smart cities using software-defined networking," in 2016 IEEE International Smart Cities Conference (ISC2), Sept 2016, pp. 1-4.

[16] (2017, October 23 2017) Global specialist in energy management and automation I schneider electric. [Online]. Available: https://www.schneider-electric.us/en/.

[17] U. EIA, “Annual energy outlook 2010," US Energy Information Administration, Washington, DC, pp. 60-62, 2013.

[18] U. AAMT, "Membrane desalination power usage put in perspective," Improving America's Waters Through Membrane Treatment and Desalting, pp. 60-62, April 2016.

[19] A. Ayang, P.-S. Ngohe-Ekam, B. Videme, and J. Temga, "Power consumption: Base stations of telecommunication in sahel zone of cameroon: Typology based on the power consumption-model and energy savings," Journal of Energy, vol. 2016, 2016. 